\title{
Clinical and biological characteristics and prognostic impact of somatic GATA2 mutations in myeloid malignancies: a single institution experience
}

(c) The Author(s) 2021

Blood Cancer Journal (2021)11:122; https://doi.org/ 10.1038/s41408-021-00517-0

\section{Dear Editor,}

The GATA2 gene, located on chromosome $3 q 21$, is a member of the GATA family of six GATA transcription factors regulating gene expression via two conserved zinc finger (ZF) domains named after their corresponding location, $\mathrm{N}$ and $\mathrm{C}$ terminal ZF domains $[1,2]$. GATA2 regulates hematopoietic stem cell proliferation and differentiation [3, 4]. It regulates mononuclear cell development, alveolar macrophage activity, and plays a role in lymphatic development $[5,6]$. Different location of GATA2 mutation is associated with distinct phenotype and leukemogenic mechanisms, with ZF-2 domain mutations common in Monocytopenia and mycobacterial infection (MonoMAC) syndrome /Dendritic Cell, Monocyte, B and NK Lymphoid deficiency syndrome (DCML), familial myelodysplastic syndrome/acute myeloid, and chronic myeloid leukemia (CML) blast transformation [7, 8]. ZF1 mutations have been described in close association with biallelic CEBPA (biCEBPA) mutations and French-American-British (FAB) M1 subtype in adult acute myeloid leukemia (AML) [9]. Nonsense or frameshift GATA2 mutations reduce the overall expression of GATA2 protein. This is in contrast to missense mutations affecting the GATA2 ZF1 and ZF2 domains, which cause loss of function by impairing binding to GATA-DNA motifs [5]. Somatic GATA2 mutation is found in $1-4 \%$ of patients with sporadic myeloid malignancies [10-12]. Greif et al. described GATA2 mutation in $40.6 \%$ of 32 cytogenetically normal biCEBPA-mutated AML patients. All of the GATA2 mutations were missense and located in the ZF-1 domain $[13,14]$. Another study reported that most of GATA2 mutations (66.7\%) in biCEBPA AML occurred in the ZF1 domain [15].

We hypothesize that the GATA2 mutation location and mutation type have distinct impact on clinical and biological features of myeloid neoplasms ( $\mathrm{MN})$, along with their interaction with other co-occurring somatic mutations. We retrospectively analyzed (after approval by our institutional review board) 3872 consecutive patients who had next-generation sequencing (NGS) testing between 5/2015 and 7/2020 at Mayo Clinic, and identified fiftyfour MN patients harboring 63 GATA2 mutations (Supplementary materials and methods). Unlike most somatic GATA2 studies (limited to $A M L)$, our study included four different diagnostic groups; $15(27.7 \%)$ with myelodysplastic syndromes (MDS), 16 (29.6\%) with myelodysplastic/myeloproliferative neoplasms (MDS/ MPN), 9 (16.6\%) with myeloproliferative neoplasms (MPN), and 14 $(25.9 \%)$ AML (Table 1$)$. The median age was 67 years (range, 26-89) and 36 (67\%) patients were males. The median white blood cell (WBC) was $12.9 \times 10^{9} / \mathrm{L}$, median hemoglobin $(\mathrm{Hg})$ of $8.6 \mathrm{~g} / \mathrm{dl}$ and platelets of $53 \times 10^{9} / \mathrm{L}$, respectively (Table 1 and Supplemental Table S1).

The median variant allele frequency (VAF) of GATA2 mutations was $31 \%$ (range, $5-74 \%$ ), with a median VAF of $28,37,34$, and $39 \%$ in MDS, MDS/MPN, MPN, and AML, respectively. There was no statistically significant difference between median VAF in the different diagnostic groups $(p=0.6)$. Seven patients harbored two, and one patient had three GATA2 mutations. The most frequent variants were p.Lys390del $(N=5,9 \%)$, p.Met388_Lys389del $(N=4$, $8 \%)$ and p.Arg396Trp $(N=3,6 \%)$, while other variants were detected in 1-2 patients (Fig. $1 \mathrm{~A}$ and Supplemental Table S2). Thirty-one (57\%) mutations clustered in ZF2, 9 (17\%) in the ZF1 domain, and 14 (26\%) were outside the ZF domains (non-ZF). ZF1 and non-ZF mutated patients had lower mean VAF compared to ZF2 patients ( $22 \%$ vs. $38 \%, p=0.03)$ and $(26 \%$ vs. $38 \%, p=0.02)$, respectively. The most common GATA2 mutation types were missense mutation $(N=18,33.3 \%)$ and in-frame deletion $(N=18$, 33.3\%). Other less common mutation types were frameshift $(N=$ $12,22.2 \%)$, nonsense $(N=4,7.4 \%)$, and duplication that span through splicing site $(N=2,3.7 \%)$ (Supplemental Table S2). Duplication that spans through splicing site, in-frame deletion, and nonsense mutations occurred only in ZF1, ZF2, and non-ZF, respectively. ZF1 patients had significantly higher frequency of missense mutations compared to ZF2 patients (78\% vs. $35 \%, p=$ 0.02). Frameshift mutation was more common in non-ZF patients compared to ZF2 patients ( $83 \%$ vs. $17 \%, p<0.0001$ ). AML patients had significantly more ZF1 mutations compared to chronic MN ( $43 \%$ vs. $7.5 \%, p=0.002$ ). MPN patients harbored significantly more frequent ZF2 mutations and in-frame deletions compared to other diagnostic groups ( $89 \%$ vs. $51 \%, p=0.003)$ and $(67 \%$ vs. $27 \%, p=0.003)$, respectively. Thirty-two $(63 \%)$ patients showed cytogenetic abnormalities by karyotyping while 19 (37\%) patients were normal. The most common cytogenetic abnormalities involved chromosome 7 (3 of which were monosomy7) abnormalities $(N=9,28 \%)$, trisomy $8(N=7,22 \%)$, and 20 q deletion $(N=$ 6, 19\%). (Supplemental Table S3).

We then investigated the interaction of 28 distinct co-mutations in 52 (96\%) patients. ASXL1 (60\%), SRSF2 (33\%), RUNX1 (19\%), U2AF1 (59\%), TET2 (17\%), and SF3B1 (13\%) were the most common co-mutations (Supplemental Fig. S1, and Supplemental Table S48). Median number of mutations per patient was 4 (range $=1-6$ ) (Supplemental Table S9). Thirty-four, 32, 24, 15, and 13 patients had at least one mutation in chromatin modification, RNA splicing, signaling, DNA methylation, and transcription pathway, respectively (Supplemental Table S10). GATA2-ZF2 and non-ZF had significantly higher frequency of $A S X L 1$ co-mutation compared to GATA2-ZF1 (74\% vs. $11 \%, p=0.0007)$ and (50\% vs. $11 \%, p=0.04)$, respectively. Chronic MN had significantly higher frequency of 
Table 1. Patients characteristics with GATA2 mutation.

a. Characteristics and hematological features of patients with GATA2 mutation

\begin{tabular}{|c|c|}
\hline Variable & Value \\
\hline No. of patients & 54 \\
\hline Age years, median(range) & $67(26-89$ \\
\hline Sex (male), $n(\%)$ & $36(67)$ \\
\hline Hemoglobin G/DL, median (range) & $8.6(5.8-1)$ \\
\hline Leukocytes $10^{9} / \mathrm{L}$, median (range) & $12.9(0.3-$ \\
\hline Thrombocytes $10^{9} / \mathrm{L}$, median (range) & $53(1.5-8)$ \\
\hline MCV median (range) & $93(77-11$ \\
\hline ANC, median (range) & $6.64(0.17$ \\
\hline AMC, median (range) & $0.39(0-4$ \\
\hline ALC, median (range) & $1.44(0.37$ \\
\hline RDW, median (range) & $17.7(13.1$ \\
\hline Number of mutations, median (range) & $4(1-6)$ \\
\hline \multicolumn{2}{|l|}{ Diagnosis } \\
\hline MDS, $n(\%)$ & $15(27.7)$ \\
\hline MPN, $n(\%)$ & $9(16.6)$ \\
\hline AML, $n(\%)$ & $14(25.9)$ \\
\hline MDS/MPN, $n(\%)$ & $16(29.6)$ \\
\hline \multicolumn{2}{|l|}{ Mutation types } \\
\hline Missense, $n(\%)$ & $18(33.3)$ \\
\hline In-frame deletion, $n$ (\%) & $18(33.3)$ \\
\hline Frameshift, $n(\%)$ & $12(22.2)$ \\
\hline Non-sense, $n(\%)$ & $4(7.4)$ \\
\hline Duplication that span through splicing site, $n(\%)$ & $2(3.7)$ \\
\hline \multicolumn{2}{|l|}{ Mutation location } \\
\hline $\mathrm{ZF} 1, n(\%)$ & $9(17)$ \\
\hline $\mathrm{ZF} 2, n(\%)$ & $31(57)$ \\
\hline Non-ZF, $n(\%)$ & $14(26)$ \\
\hline \multicolumn{2}{|l|}{ Abnormal cytogenetics } \\
\hline Yes, $n(\%)$ & $32(63)$ \\
\hline No, $n(\%)$ & $19(37)$ \\
\hline
\end{tabular}

b. Summary of molecular findings according to GATA2 mutation location.

\begin{tabular}{|c|c|c|c|}
\hline Location & ASXL1 co-mutations (\%) & Mutation type (\%) & Unique co-mutations \\
\hline ZF-1 & 11 & $\begin{array}{l}\text { Missense }(78 \%) \text {, duplication that span through } \\
\text { splicing site }(22 \%)\end{array}$ & CEBPA, KIT, SF3B1, TET2 \\
\hline ZF-2 & 74 & $\begin{array}{l}\text { In-frame deletion (58\%), missense }(35.5 \%), \\
\text { frameshift }(6.5 \%)\end{array}$ & $J A K 2$ \\
\hline Non-ZF & 50 & Frameshift $(71 \%)$, non-sense ( $29 \%)$ & None \\
\hline
\end{tabular}

c. Summary of molecular findings according to the diagnostic groups.

\begin{tabular}{|c|c|c|c|c|c|}
\hline Diagnosis & $\begin{array}{l}\text { GATA2 mutation } \\
\text { location }(\%)\end{array}$ & $\begin{array}{l}\text { Most frequent GATA2 } \\
\text { mutation type (\%) }\end{array}$ & $\begin{array}{l}\text { Frequency of } A S X L 1 \text { co- } \\
\text { mutations (\%) }\end{array}$ & $\begin{array}{l}\text { Unique co- } \\
\text { mutations }\end{array}$ & $\begin{array}{l}\text { Patients with several } \\
\text { GATA2 mutations (N) }\end{array}$ \\
\hline AML & $\begin{array}{l}\text { ZF-1 (43\%), ZF-2 } \\
(36 \%), \text { Non-ZF (21\%) }\end{array}$ & Missense (50\%) & 14 & $\begin{array}{l}\text { CEBPA KIT } \\
\text { FLT3 }\end{array}$ & 1 \\
\hline
\end{tabular}

$M C V$ mean corpuscular volume, $A N C$ absolute neutrophil count, $A M C$ absolute monocyte count, $A L C$ absolute lymphocyte count, $R D W$ red cell distribution width, MDS myelodysplasia neoplasm, AML acute myeloid leukemia, MPN Myeloproliferative neoplasms, MDS/MPN Myelodysplastic/Myeloproliferative neoplasms, ZF zing finger. 
A

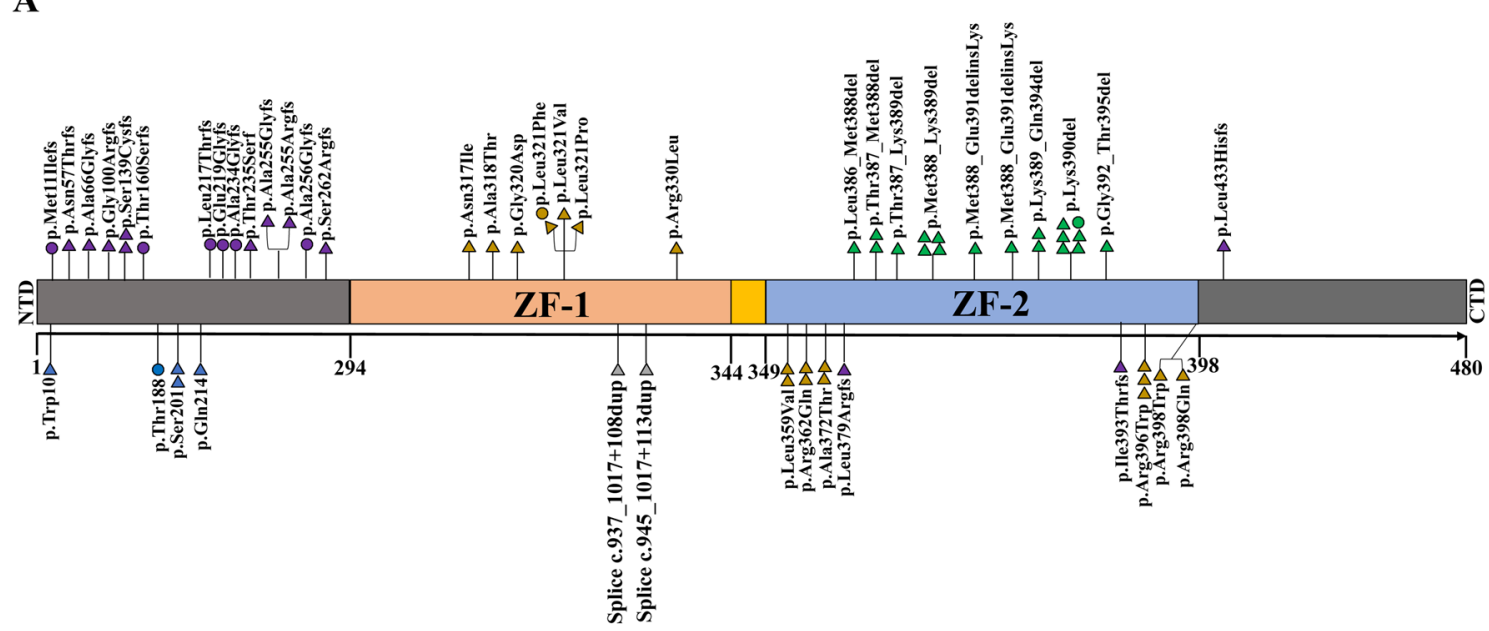

- Frameshift ${ }^{-}$Missense ${ }^{-}$In-frame deletion ${ }^{-}$Nonsense ${ }^{-}$Duplication span through splicing site

○ Second $G A T A 2$ mutation

B

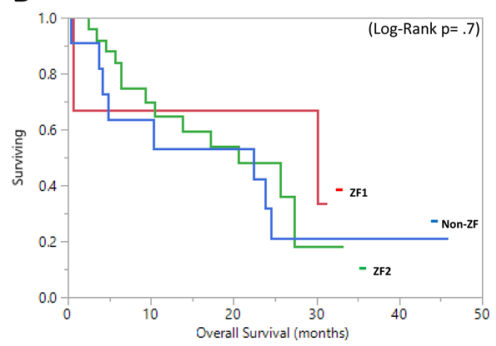

C

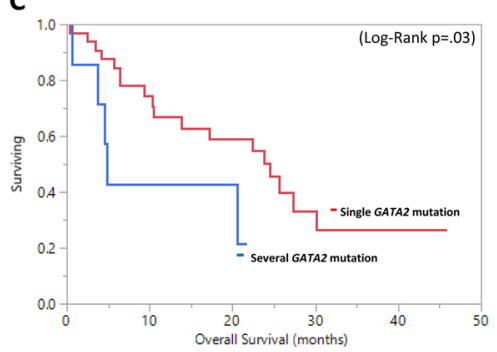

D

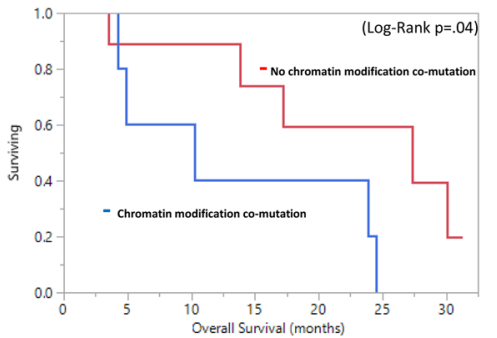

Fig. 1 GATA2 gen mutations distribution and survival outcome in myeloid neoplasms. A Representation of GATA2 variants detected, positioned on the GATA2 protein, and its functional domains. NTD N-terminal domain, ZF zinc finger domain, CTD C-terminal domain. Kaplan-Meier survival curves for OS B Stratified by GATA2 mutation location in 40 chronic myeloid neoplasm patients. There was no difference in median OS between GATA2-ZF1, ZF2, and non-ZF mutations (30 vs. 20.6 vs. 22.4 months, $p=0.7$ ). C Stratified by the number of GATA2 mutations in 40 chronic myeloid neoplasm patients. D Stratified by chromatin modification co-mutation status in 15 MDS patients.

ASXL1 co-mutation compared to AML (73\% vs. $14 \%, p=0.0001)$. Mean age of GATA2-mutated patients with ASXL1 mutations was higher than that of patients without an ASXL1 mutation (70 vs. 62 years, $p=0.02$ ).

Two biCEBPA and two KIT mutations occurred in ZF1-mutated patients, but none were co-mutated in non ZF-1 mutated patients. $B I C E B P A, K I T$, and FLT3 were co-mutated only in AML but not in chronic MN. ZF1-mutated patients had a significantly higher frequency of SF3B1 (44\% vs. 7\%, $p=0.002$ ) and TET2 co-mutations ( $56 \%$ vs. $9 \%, p=0.0006$ ) than non ZF-1. ZF2-mutated patients had a significantly higher frequency of JAK2 (19\% vs. $0 \%, p=0.02)$ than non ZF-2 patients.

GATA2-mutated MPN patients had a significantly higher frequency of CALR, MPL, and JAK2 co-mutation (22\% vs. $0 \%$, $p=0.001),(11 \%$ vs. $0 \%, p==0.02),(33 \%$ vs. $6.7 \%, p=0.02)$ than other MN. GATA2-mutated MDS patients had a significantly higher frequency of $B C O R(20 \%$ vs. $3 \%, p=0.02)$, and U2AF1 (33\% vs. $10 \%, p=0.04)$ co-mutations, whereas MDS/MPN overlap patients showed a significantly higher frequency of ASXL1, SETBP1, and SRSF2 co-mutation (94\% vs. $42 \%, p=0.0005),(13 \%$ vs. $0 \%$, $p=0.02)$, (56\% vs. $21 \%, p=0.01)$ than other MN.

In-frame deletion GATA2 mutation co-occurred with ASXL1, $S R S F 2$, and ZRSR2 mutations at a higher frequency of ( $89 \%$ vs. $42 \%$, $p=0.0009),(50 \%$ vs. $22 \%, p=0.03),(11 \%$ vs. $0 \%, p=0.04)$ compared to other mutation types. GATA2 missense mutation, nonsense mutation, and frameshift mutation had higher frequency of SF3B1 (28\% vs. $6 \%, p=0.02), E Z H 2$ (50\% vs. $6 \%$, $p=0.003)$, and SETBP1 $(17 \%$ vs. $0 \%, p=0.007)$ co-mutation compared to other mutation types, respectively.

Overall, 34 (63\%) patients died after a median follow-up period of 26.4 months. The median overall survival (mOS) was 13.8 months for the overall cohort, and 23.9, 22.4, 20.6, and 5.6 months in MDS, MDS/MPN, MPN, and AML patients, respectively. There was no statistically significant difference in survival between diagnostic groups $(p=0.1)$. After excluding AML patients for OS analysis, we found that the 7 patients harboring more than one GATA2 mutations had worse mOS (4.9 vs. 24.5 months, $p=0.03)(\mathrm{HR}=$ $3.01, p=0.04$ ) compared to those with single GATA2 mutation (Fig. 1). Patients with chromatin modification co-mutation had significantly worse 1 -year estimated OS (1-yr-OS) (57\% vs. $86 \%, p=$ 0.048). Multivariate analysis demonstrated that co-mutation in chromatin modification and multi-GATA2 mutations were independent poor prognostic factors for OS with HR of $4.8(p=0.047)$ and HR of $3.5(p=0.02)$, respectively (Supplemental Table S11) in chronic MN. Similarly, MDS patients with chromatin modification co-mutation had worse mOS than their un-mutated counterparts (17 vs. 30 months, $p=0.04$, Fig. 1). MDS patients with frameshift (FS) mutation (10.3 vs. 27.37 months, $p=0.048$ ) and in-frame deletion mutation ( 8.7 vs. 24.5 months, $p=0.03$ ) had worse mOS than non-FS mutation and non-in-frame deletion, respectively (Supplemental Fig. S2). There was no significant difference in OS in regards to mutation type in other diagnostic groups. MDS/MPN 
overlap patients harboring RUNX1 co-mutation had worse mOS ( 2.2 vs. 25.7 months, $p=0.0004)$ compared to those without the comutation. Finally, although Tien et al. found that ZF1-mutated AML patients had better OS than GATA2 ZF2-mutated patients, we could not find such difference in non-AML patients with mOS in GATA2$\mathrm{ZF1}$, ZF2, and non-ZF (30 vs. 20.6 vs. 22.4 months, $p=0.7$ ), respectively (Fig. 1) [9]. Seven out of 40 (17.5\%) chronic MN patients progressed into AML with a median leukemic-free survival of 10.4 months.

Our study is limited due to the small sample size, retrospective nature, and inability to rule out the possibility of germline mutation, although the clinical and family history and/ or the mutational VAF in these cases did not support a germline nature. We, for the first time, were able to investigate the interaction of different ZF domain mutations with other somatic mutations that recurrently occur in myeloid neoplasms. Our findings confirm the higher frequency of ZF1 with AML and missense mutation. In addition, they suggest that ASXL1 mutations co-occur more frequently with chronic MN and ZF2-GATA2 mutations than AML and ZF-1-GATA2 mutations, respectively. GATA2 in-frame deletions and JAK2 co-mutation occurred exclusively in ZF-2-GATA2-mutated cases, whereas non-sense mutations and duplications that span through the splicing site occurred in non-ZF and ZF-1 regions, respectively. We propose that larger cohorts are needed to validate our findings and further explore the association and implication of different somatic GATA2 mutations.

Ahmad Nanaa (D) ${ }^{1}$, David Viswanatha², Zhuoer Xie (D) ${ }^{1}$, Dragan Jevremovic (iD ${ }^{2}$, Phuong Nguyen ${ }^{2}$, Mohamad E. Salama ${ }^{2}$, Patricia Greipp ${ }^{3}$, Kurt Bessonen ${ }^{2}$, Naseema Gangat ${ }^{1}{ }_{1}^{1}$, Mrinal Patnaik $\mathbb{D}^{1}$, Animesh Pardanani ${ }^{1}$, Hassan B. Alkhateeb ${ }^{1}{ }^{1}$ Mithun Shah (iD ${ }^{1}$, William Hogan (iD ${ }^{1}$, Ayalew Tefferi (D) ${ }^{1}$ Mark Litzow (D) ${ }^{1}$, Rong He (ID) ${ }^{2 \bowtie}$ and Aref Al-Kali (D) ${ }^{7}$ Division of Hematology, Mayo Clinic, 200 First Street SW, Rochester, MN 55905, USA. ${ }^{2}$ Division of Hematopathology, Mayo Clinic, 200 First Street SW, Rochester, MN 55905, USA. ${ }^{3}$ Division of Cytogenetics, Mayo Clinic, 200 First Street SW, Rochester, MN 55905, USA. 凶email: he.rong@mayo.edu; alkali.aref@mayo.edu

\section{REFERENCES}

1. Orkin SH. Diversification of haematopoietic stem cells to specific lineages. Nat Rev Genet. 2000;1:57-64.

2. Rodrigues NP, Tipping AJ, Wang Z, Enver T. GATA-2 mediated regulation of normal hematopoietic stem/progenitor cell function, myelodysplasia and myeloid leukemia. Int J Biochem Cell Biol. 2012;44:457-60.

3. Tsai F-Y, Keller G, Kuo FC, Weiss M, Chen J, Rosenblatt $M$, et al. An early haematopoietic defect in mice lacking the transcription factor GATA-2. Nature. 1994;371:221-6.

4. Tsai FY, Orkin SH. Transcription factor GATA-2 is required for proliferation/survival of early hematopoietic cells and mast cell formation, but not for erythroid and myeloid terminal differentiation. Blood. 1997;89:3636-43.

5. Collin M, Dickinson R, Bigley V. Haematopoietic and immune defects associated with GATA2 mutation. Br J Haematol. 2015;169:173-87.

6. Kazenwadel J, Secker GA, Liu YJ, Rosenfeld JA, Wildin RS, Cuellar-Rodriguez J, et al. Loss-of-function germline GATA2 mutations in patients with MDS/AML or MonoMAC syndrome and primary lymphedema reveal a key role for GATA2 in the lymphatic vasculature. Blood. 2012;119:1283-91.

7. Bresnick EH, Katsumura KR, Lee $\mathrm{H}-\mathrm{Y}$, Johnson KD, Perkins AS. Master regulatory GATA transcription factors: mechanistic principles and emerging links to hematologic malignancies. Nucleic Acids Res. 2012;40:5819-31.
8. Zhang S-J, Ma LY, Huang QH, Li G, Gu BW, Gao XD, et al. Gain-of-function mutation of GATA-2 in acute myeloid transformation of chronic myeloid leukemia. Proc Natl Acad Sci USA. 2008;105:2076-81.

9. Tien F-M, Hou HA, Tsai CH, Tang JL, Chiu YC, Chen CY, et al. GATA2 zinc finger 1 mutations are associated with distinct clinico-biological features and outcomes different from GATA2 zinc finger 2 mutations in adult acute myeloid leukemia. Blood Cancer J. 2018;8:87.

10. Lindsley RC, Mar BG, Mazzola E, Grauman PV, Shareef S, Allen SL, et al. Acute myeloid leukemia ontogeny is defined by distinct somatic mutations. Blood. 2015;125:1367-76.

11. Papaemmanuil E, Gerstung M, Bullinger L, Gaidzik Vl, Paschka P, Roberts ND, et al. Genomic classification and prognosis in acute myeloid leukemia. New Engl J Med. 2016;374:2209-21.

12. Papaemmanuil E, Gerstung M, Malcovati L, Tauro S, Gundem G, Van Loo P, et al. Clinical and biological implications of driver mutations in myelodysplastic syndromes. Blood. 2013;122:3616-27.

13. Dufour A, Konstandin N, Ksienzyk B, Zellmeier E, Benthaus T, Yaghmaie $M$, et al. High frequency of GATA2 mutations in cytogenetically normal acute myeloid leukemia with biallelic CEBPA mutations identified by exome sequencing. Blood. 2011;118:72.

14. Greif PA, Dufour A, Konstandin NP, Ksienzyk B, Zellmeier E, Tizazu B, et al. GATA2 zinc finger 1 mutations associated with biallelic CEBPA mutations define a unique genetic entity of acute myeloid leukemia. Blood. 2012;120:395-403.

15. Fasan A, Eder C, Haferlach C. GATA2 mutations are frequent in intermediate-risk karyotype AML with biallelic CEBPA mutations and are associated with favorable prognosis. Leukemia. 2013;27:482-5.

\section{AUTHOR CONTRIBUTIONS}

$A A, A N$, and $\mathrm{RH}$ designed the study; $\mathrm{AN}$ and $\mathrm{RH}$ collected and analyzed the NGS data; $\mathrm{AN}$ and AA conducted the statistical analysis and wrote the manuscript which was reviewed and edited by all authors.

\section{COMPETING INTERESTS}

The authors declare no competing interests.

\section{ADDITIONAL INFORMATION}

Supplementary information The online version contains supplementary material available at https://doi.org/10.1038/s41408-021-00517-0.

Correspondence and requests for materials should be addressed to R.H. or A.A-K.

Reprints and permission information is available at http://www.nature.com/ reprints

Publisher's note Springer Nature remains neutral with regard to jurisdictional claims in published maps and institutional affiliations.

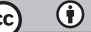

Open Access This article is licensed under a Creative Commons Attribution 4.0 International License, which permits use, sharing, adaptation, distribution and reproduction in any medium or format, as long as you give appropriate credit to the original author(s) and the source, provide a link to the Creative Commons license, and indicate if changes were made. The images or other third party material in this article are included in the article's Creative Commons license, unless indicated otherwise in a credit line to the material. If material is not included in the article's Creative Commons license and your intended use is not permitted by statutory regulation or exceeds the permitted use, you will need to obtain permission directly from the copyright holder. To view a copy of this license, visit http://creativecommons. org/licenses/by/4.0/.

(c) The Author(s) 2021 